\title{
J-chronic total occlusion score predictive capacity for percutaneous coronary intervention success of chronic total occlusion: Results from a European single center cohort with progressive experience over time
}

\author{
Mohsen Mohandes ${ }^{1}$, Cristina Moreno ${ }^{1}$, Sergio Rojas ${ }^{1}$, Victor Doblas ${ }^{1}$, \\ Mónica Fuertes ${ }^{1}$, Francisco Fernández ${ }^{1}$, Alberto Pernigotti ${ }^{1}$, Jordi Guarinos ${ }^{1}$, \\ Mercé Camprubi ${ }^{2}$, Alfredo Bardaji ${ }^{2}$ \\ ${ }^{1}$ Interventional Cardiology Unit, Cardiology Division, Joan XXIII University Hospital, \\ Pere Virgili Health Research Institute (IISPV), Tarragona, Spain \\ ${ }^{2}$ Cardiology Division, Joan XXIII University Hospital, Pere Virgili Health Research Institute (IISPV), \\ Tarragona, Spain
}

\begin{abstract}
Background: Several scoring systems have been described to assess the level of difficulty and to predict the probability of success of percutaneous coronary intervention (PCI) of chronic total occlusion (CTO). The J-CTO score was initially developed to correlate CTO complexity with guidewire time crossing through the lesion within 30 min. Moreover, almost all scoring systems represent procedures performed by seasoned operators. Herein, this study sought to evaluate the predictive capacity of J-CTO for PCI success in a European single-center cohort with growing experience in the approach of CTO.

Methods: Five hundred twenty-six procedures were performed between 2007 and 2020 mainly by a single operator. The predictive power of J-CTO score was assessed by area under the receiver-operator characteristic curve (ROC) in the entire cohort and additionally in two separate periods. The goodnessof-fit of the model was evaluated by the Hosmer and Lemeshow statistic.

Results: Successful procedure in first-attempt PCI was $79.5 \%$ and the overall success including 47 repeated procedures was achieved in $85.8 \%$. The retrograde approach was attempted in $14.4 \%$. The score was inversely associated with procedural success with lower success rate in more difficult CTOs $(p<0.001)$. ROC curve for the entire cohort, and first block (case 1-200) and second block (case 201-526) was 0.696, 0.661 and 0.748, respectively. The model showed good calibration for the entire $\operatorname{cohort}\left(X^{2}=1.7 ; p=0.43\right)$.

Conclusions: J-CTO score showed an acceptable predictive power for procedural success in this cohort although its discriminatory power is better as the level of experience is improved. (Cardiol J 2023; 30, 1: 59-67)
\end{abstract}

Key words: percutaneous coronary intervention, chronic total occlusion (CTO), J-CTO score

Address for correspondence: Mohsen Mohandes, MD, Interventional Cardiology Unit, Cardiology Division, Joan XXIII University Hospital, Pere Virgili Health Research Institute (IISPV), Calle Dr Mallafré Guasch 4, 43007 Tarragona, Spain, tel: 0034-977295817, e-mail: mohandesmohsen@hotmail.com

Received: 14.03.2021 Accepted: 15.05.2021 Early publication date: 9.06.2021

This article is available in open access under Creative Common Attribution-Non-Commercial-No Derivatives 4.0 International (CC BY-NC-ND 4.0) license, allowing to download articles and share them with others as long as they credit the authors and the publisher, but without permission to change them in any way or use them commercially. 


\section{Introduction}

Percutaneous coronary intervention (PCI) of chronic total occlusion (CTO) has been regarded as one of the most challenging coronary intervention procedures. Although the incorporation of new and specific devices has dramatically increased procedural success [1], it is still lower than that of PCI of other complex non-occluded arteries [2].

Although successful recanalization of a CTO compared with optimal medical therapy in patients with stable angina has been associated with better symptoms control and quality of life [3], the treatment of these kinds of lesions are more time consuming and technically demanding. Therefore, as a complex category of coronary intervention, the design of a proper strategy and evaluation of the case complexity before treating a CTO is crucial. To address this issue several scores, based essentially on anatomical features of the CTO, have been described during the last decade in an attempt to try to categorize the lesion complexity and the likelihood of procedural success [4-6]. Among several CTO scores presented so far, the $\mathrm{J}$-CTO [7] is one of the simplest and most practical and probably the most widely used in many interventional cardiology units nowadays. However, J-CTO score was initially described to estimate the successful guidewire passage of CTO body within 30 min depending, on various anatomic features such as in-CTO segment tortuosity, CTO segment length, calcification, blunt stump type as well as previous failed attempt. Although wire crossing is a key step for final success in a CTO-PCI, it is not synonymous. Though some studies have validated the ability of J-CTO score to predict the PCI final success, others have failed to confirm its predictability [8].

Besides, almost all studies presenting different scores for the CTO approach were comprised of procedures reportedly performed by experienced operators. The learning curve in the subset of CTO-PCI is something that seems to be a definitive key factor associated with guide wire crossing and procedural success [9].

This study sought to evaluate the ability of J-CTO score to predict the PCI success rate in a European single center cohort with growing experience since the installation of a CTO-PCI program in the institution. The applicability and usefulness of this simple score in such a scenario is something we intended to examine closely.

\section{Methods}

We started a CTO program in our institution in May 2007 with a low case volume during its initial stages increasing trend in patient recruitment with at least 50 procedures a year since 2013 . Five workshops over 5 consecutive years with experienced operators were organized in our center as part of training in CTO-PCI and in order to improve local operators' experience. We had a dedicated operator in our institution for CTOs, although a few procedures were performed by a second operator in the first 100 block of cases. A CTO was defined as the presence of a coronary artery segment obstruction greater than 3 months standing with Thrombolysis in Myocardial Infarction (TIMI) grade 0 flow [10]. All data were prospectively introduced into a database including all potential angiographic variables previously related to the difficulty of the procedural success such as ostial location for example [11]. Since the publication of the J-CTO score by Morino et al. [7] in 2011 derived from Multicenter CTO Registry in Japan [12] those variables related to the wire crossing time through a CTO in this study were introduced in our database and reviewed and checked retrospectively. The J-CTO score was derived from the analysis of a cohort of 494 CTO-PCIs defining the complexity of the cases in accordance with the guidewire crossing through the CTO segment within 30 min [7]. Five independent predictors were found: in segment or CTO entry tortuosity more than $45^{\circ}$, calcification, the CTO length $\geq 20 \mathrm{~mm}$, a blunt stump and any reattempted procedure. The score gives one point to each variable, if present, categorizing the level of difficulty of the procedure in easy $(\mathrm{J}-\mathrm{CTO}=0)$, intermediate $(\mathrm{J}-\mathrm{CTO}=1)$, difficult $(\mathrm{J}-\mathrm{CTO}=2)$ and very difficult $(\mathrm{J}-\mathrm{CTO} \geq 3$ ), respectively. A thorough review of the above-mentioned variables was carried out by two observers working in our cath lab and, in case of any discrepancy the opinion of a third examiner was asked and a final consensus was established. After completing the review, J-CTO variables of 50 randomly selected cases were examined again and the level of concordance between two observers was estimated in order to assess and resolve any possible interobserver bias. Successful angiographic result was defined when recanalization of the occluded artery with final TIMI flow grade III (TIMI III) and residual lesion less than $30 \%$ was achieved. All complication such as in-hospital death, peri-procedural myocardial infarction (MI), coronary perforation requiring 
pericardiocentesis, major vascular complications needing percutaneous or surgical intervention were reported and its incidence was compared between failed and successful procedure groups. Attribution of peri-procedural MI was in accordance with the universal definition of PCI-related MI (type 4a) [13].

\section{Details of the procedures}

Contralateral injection using double access was used in case any hetero collateral was found in the diagnostic angiogram. Dedicated wires and microcatheters for crossing the CTO segment were used in all procedures and the segment before the occlusion was traversed with a floppy wire in order to minimize any damage to the artery. Anterograde approach was the preferred strategy during the initial steps of our learning curve and retrograde approach was incorporated later and was used either after failing the antegrade approach or as an initial strategy. In all cases activated clothing time was checked every 30 min to keep a level of 250-300 s for antegrade and between 300-350 s for retrograde access.

\section{Statistical analysis}

Continuous variables were presented as mean \pm standard deviation. Categorical variables were expressed as absolute numbers and percentages. The Student $t$ statistic was used to compare quantitative variables between two groups and $\chi^{2}$ test was applied to evaluate the association between qualitative variables. The level of interobserver concordance regarding the measurement of J-CTO variables was assessed by Kappa index statistic. Multivariate model was constructed with variables included in J-CTO score and the predictive capacity of the model was determined with multivariate logistic regression. The goodness-of-fit of the model was assessed with Hosmer and Lemeshow (HL) test so as to evaluate any possible discrepancy between observed and expected values. Subsequently the discriminatory power of the logistic model was estimated by the area under the receiver operator characteristic curve (ROC) or C/index. An additional analysis of two periods represented by cases 1 to 200 (first block) and cases 201 to 526 (second block) was performed in order to assess the potential influence of learning curve in modification of the predictive capacity of the model.

All analyses were performed using the statistical package of SPSS 19 and the $p$ value of $<0.05$ was considered statistically significant.

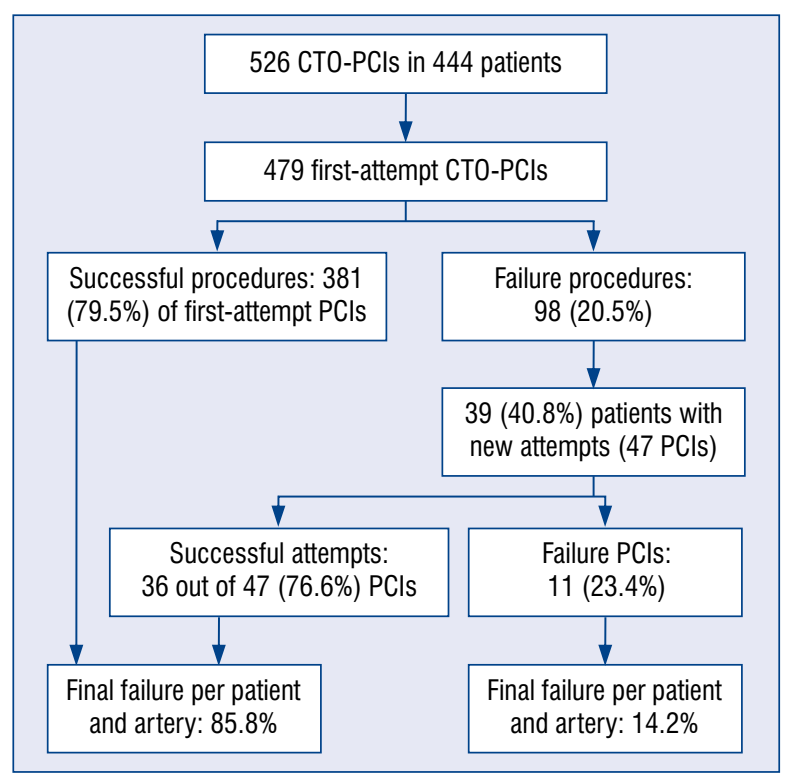

Figure 1. Flowchart of patients and percutaneous coronary intervention $(\mathrm{PCl})$ in the entire cohort and procedural result in different steps; СTO - chronic total occlusion.

\section{Results}

A total of 526 of CTO-PCIs in 444 patients between May 2007 and November 2020 were performed in our cath lab (Fig. 1). From May 2007 up to December 2012, 123 procedures were carried out and in the second period up to November 2020, the remaining $403 \mathrm{PCIs}$ were done with an average of 50 cases per year. The mean age of overall population was $65.2 \pm 10.9$ and $81(15.4 \%)$ of the patients were women. The prevalence of multivessel disease in this series was 334 (63.5\%) and $77(14.6 \%)$ of cases had left ventricular ejection fraction less than $40 \%$ (Table 1 ).

The level of interobserver concordance for the estimation of J-CTO variables assessed by Kappa index was 0.8 .

First-attempt procedural success was achieved in $381(79.5 \%)$. Thirty-nine patients had repeated attempts on the same artery after failing the first procedure. One out of 39 patients had 2 CTOs, each of them attempted twice. One patient had 2 CTOs, one of them was tried twice and the other attempted 4 times; and, 1 patient had 1 CTO that was tried 4 times. The remaining patients had 1 CTO attempted twice. The overall repeated procedures were 47 PCIs. Thirty-six out of 47 (76.6\%) reattempted CTO-PCIs were successful resulting 
Table 1. Baseline characteristics of the overall population $(n=526)$.

\begin{tabular}{lc}
\hline Age [years] & $65.2 \pm 10.9$ \\
Female & $81(15.4 \%)$ \\
$\mathrm{BMI}\left[\mathrm{kg} / \mathrm{m}^{2}\right]$ & $28.8(4.7 \%)$ \\
GFR & $87.3(35.2 \%)$ \\
Diabetes & $239(45.4 \%)$ \\
Hypertension & $395(75.1 \%)$ \\
Dyslipidemia & $374(71.1 \%)$ \\
Smoking & $313(59.5 \%)$ \\
Previous MI & $239(45.4 \%)$ \\
Previous CABG & $42(8 \%)$ \\
LVEF < 40\% & $77(14.6 \%)$ \\
Stable angina & $350(66.5 \%)$ \\
NSTEMI & $148(26.6 \%)$ \\
Heart failure & $36(6.8 \%)$ \\
Multivessel disease & $334(63.55 \%)$ \\
\hline
\end{tabular}

$\mathrm{BMI}$ - body mass index; CABG — coronary artery bypass grafting; GRR - glomerular filtration rate; LVEF — left ventricular ejection fraction; $\mathrm{Ml}$ - myocardial infarction; NSTEMI - non-ST-segment elevation myocardial infarction

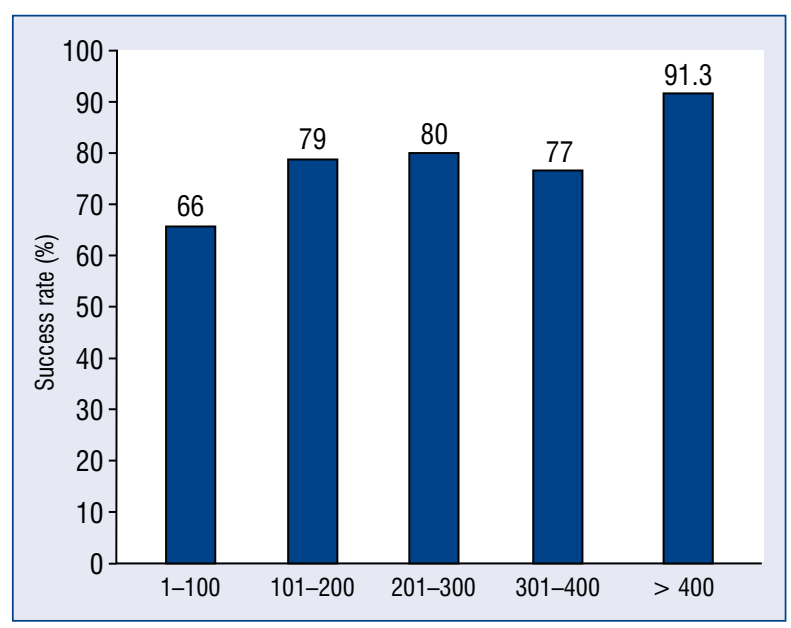

Figure 2. Procedural success rate corresponding to different blocks of cases $(1-100,101-200,201-300,301-400$ and $>400$ ( $p$ of linear trend $<0.001$ ).

in an overall PCI success rate per patient and artery of $85.8 \%$ in the entire cohort (Fig. 1). Success rate increased over time and showed a significant linear trend ( $<<0.001$; Fig. 2).

Right coronary artery (RCA) was the most treated accounting for $242(46 \%)$ of cases followed by left anterior descending coronary artery (LAD) in $174(33.1 \%)$, left circumflex artery (LCX) in 96 (18.3\%), diagonal (DG) in 7 (1.3\%), ramus inter-
Table 2. Angiographic and procedural characteristics of the cohort.

\begin{tabular}{lc}
\hline & $\begin{array}{c}\text { Study population } \\
\text { (n = 526) }\end{array}$ \\
\hline CTO site & $242(46 \%)$ \\
RCA & $174(33.1 \%)$ \\
LAD & $96(18.3 \%)$ \\
LCX & $7(1.3 \%)$ \\
DG & $3(0.6 \%)$ \\
RI & $2(0.4 \%)$ \\
LM & $2(0.4 \%)$ \\
SVG & $172(32.7 \%)$ \\
Blunt stump & $225(42.8 \%)$ \\
Calcification & $122(23.2 \%)$ \\
Tortuosity & $125(23.8 \%)$ \\
Occlusion length $\geq 20$ mm & $47(8.9 \%)$ \\
Previously failed attempt & $45(8.6 \%)$ \\
Ostial location & \\
J-CTO score & $142(27 \%)$ \\
Easy & $184(35 \%)$ \\
Intemediate & $114(21.7 \%)$ \\
Difficult & $85(16.2 \%)$ \\
Very difficult & $76(14.4 \%)$ \\
Retrograde approach & $8(1.52 \%)$ \\
ADR &
\end{tabular}

ADR - antegrade dissection reentry; CTO — chronic total occlusion; DG - diagonal; LAD - left anterior descending coronary artery; LCX - left cirumflex artery; LM — left main; RCA — right coronary artery; RI — ramus intermedius; SVG — saphenous vein graft

medius (RI) artery in $3(0.6 \%)$, left main (LM) in $2(0.4 \%)$ and saphenous vein graft (SVG) in $2(0.4 \%)$.

The level of difficulty according to J-CTO score in the cohort was represented by 142 (27\%) easy, $184(35 \%)$ intermediate, 114 (21.7\%) difficult and 85 (16.2\%) very difficult CTOs. Retrograde approach was attempted in $76(14.4 \%)$ and antegrade dissection reentry accounted for $8(1.52 \%)$ in the overall cohort (Table 2). Successful angiographic result was achieved in 128 out of $142(90.1 \%)$ in easy score group, 160 from $184(87 \%)$ in intermediate, 80 out of $114(70.2 \%)$ in difficult and in 48 of $85(56.5 \%)$ in very difficult group respectively $\left(\mathrm{X}^{2}=49.4\right.$, $\mathrm{p}<0.001$; $\mathrm{p}$ for linear trend $<0.001$; Fig. 3 ).

Failed procedures showed significant higher J-CTO score compared to successful procedures $(1.94 \pm 1.18$ vs. $1.15 \pm 1.04)$. Procedure time, fluoroscopy time and contrast medium were significantly higher in the unsuccessful than in the successful group. In terms of complications perforation requiring pericardiocentesis was experienced in 


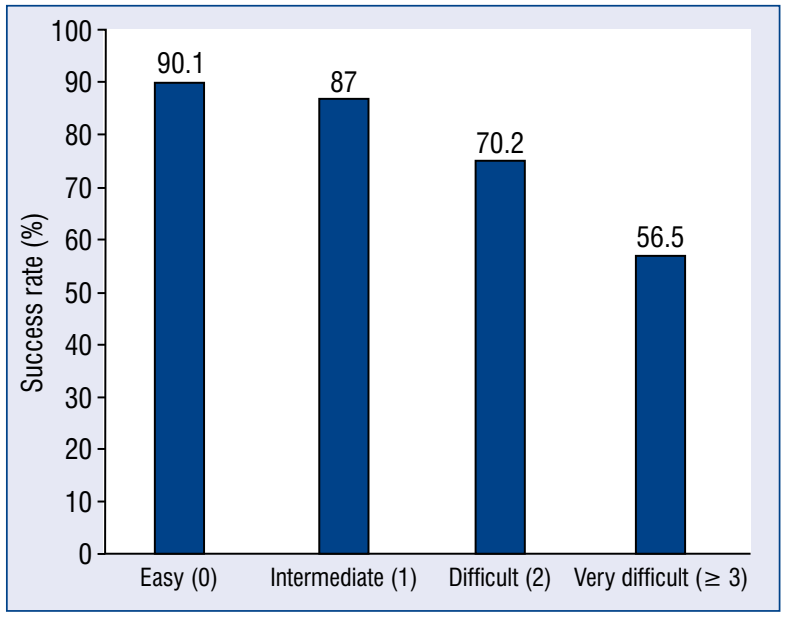

Figure 3. Procedural success rate according to different grades of J-CTO in the entire cohort; $X^{2}=49.4$; $p<0.001 ; p$ for trend $<0.001$.

$3(2.8 \%)$ in the failed group and none in the successful group and peri-procedural MI were registered in $7(6.4 \%)$ in the failure group vs. $4(0.96 \%)$ in the successful group which supposed a statistical difference in both. In-hospital death and major vascular complication did not differ significantly between groups (Table 3 ).

Success rate comparison between the first block of PCIs (1-200) and the second block (201-526) according to J-CTO score showed a significant difference $(73 \%$ vs. $83.4 \%$; $p=0.004)$ despite treating a progressive more difficult cases over time (J-CTO score: $1.15 \pm 0.95$ vs. $1.4 \pm 1.2$ respectively; $\mathrm{p}=0.01$; Table 4).

The discriminatory power of the model for the entire cohort determined by ROC curve was 0.696 (95\% confidence interval [CI] 0.639-0.752; $\mathrm{p}<0.001)$. Calibration of observed against expected rates of procedural success for different score strata as assessed by $\mathrm{HL}$ test was adequate $\left(\mathrm{X}^{2}=1.7\right.$; $p=0.43$; Fig. 4). The analysis of 200 first cases separately against the second block (case 201 to 506) showed a moderate discriminatory power for the first and good predictive capacity for the second block (C statistic: $0.661,95 \%$ CI $0.573-0.748$; $\mathrm{p}=0.001$ vs. $0.748,95 \%$ CI $0.677-0.82 ; \mathrm{p}<0.001$; Fig. 5).

\section{Discussion}

This study reveals an acceptable predictive capacity of the J-CTO score for final success rate of CTO-PCI in a European single center cohort with accumulative experience since its initial stages of the CTO program. The discriminatory power of the model performed moderately for a success rate prediction during the first period and showed a good predictive capacity for the second period.

Table 3. Comparison of baseline and angiographic characteristics and outcome between successful and failed procedures.

\begin{tabular}{lccc}
\hline & Successful procedure (n = 418) & Failed procedure (n = 108) & P \\
\hline Age [years] & $65.5 \pm 11$ & $64.2 \pm 10$ & 0.27 \\
Female & $70(16.7 \%)$ & $11(10.1 \%)$ & 0.09 \\
Hypertension & $312(74.6 \%)$ & $83(76.9 \%)$ & 0.64 \\
Diabetes & $185(44.3 \%)$ & $54(50 \%)$ & 0.29 \\
Dyslipidemia & $291(69.6 \%)$ & $83(76.9 \%)$ & 0.14 \\
Smoking & $242(57.9 \%)$ & $71(65.7 \%)$ & 0.14 \\
Previous MI & $186(44.5 \%)$ & $53(49.1 \%)$ & 0.39 \\
Previous CABG & $30(7.2 \%)$ & $12(11.1 \%)$ & 0.18 \\
Ejection fraction $<40 \%$ & $62(14.8 \%)$ & $15(13.9 \%)$ & 0.8 \\
J-CTO score & $1.15 \pm 1.04$ & $1.94 \pm 1.18$ & $<0.001$ \\
Procedure time [min] & $143.2 \pm 70.2$ & $198.3 \pm 75.5$ & $<0.001$ \\
Fluoroscopy time [min] & $63.9 \pm 42.2$ & $103.7 \pm 42.4$ & $<0.001$ \\
Contrast medium & $250 \pm 91.4$ & $272.4 \pm 92.5$ & 0.026 \\
In-hospital death & $2(0.5 \%)$ & $1(0.9 \%)$ & 0.59 \\
Perforation & $0(0 \%)$ & $3(2.8 \%)$ & 0.001 \\
Major vascular complication & $3(0.7 \%)$ & $0(0 \%)$ & $\mathrm{NS}$ \\
MI & $4(0.96 \%)$ & $7(6.4 \%)$ & 0.002 \\
\hline
\end{tabular}

Data are mean \pm standard deviation or number (percentage); CABG — coronary artery bypass graft; $\mathrm{MI}$ - myocardial infarction 
Table 4. Comparison of J-chronic total occlusion (CTO) score and percutaneous coronary intervention success rate between the first block (case 1-200) and the second block (case 201-526).

\begin{tabular}{lccc}
\hline & First block (1-200) & Second block (201-526) & P \\
\hline J-CTO score & $1.15 \pm 0.95$ & $1.4 \pm 1.2$ & 0.01 \\
J-CTO $\geq 3$ & $16(8 \%)$ & $67(20.6 \%)$ & $<0.001$ \\
Success rate & $146(73 \%)$ & $272(83.4 \%)$ & 0.004 \\
\hline
\end{tabular}

Data are mean \pm standard deviation or number (percentage).

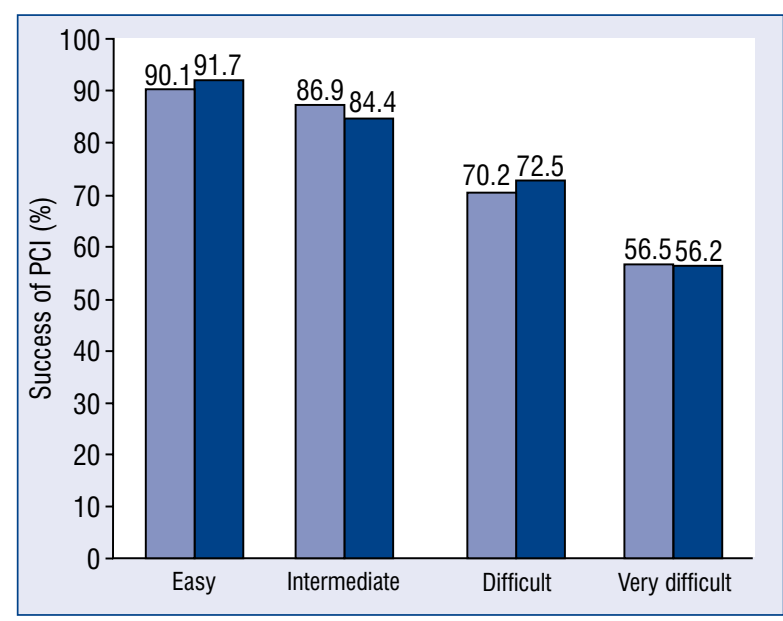

Figure 4. The result of observed and expected success rate according to J-CTO score in the entire population; Hosmer and Lemeshow $(\mathrm{HL})$ test: $\mathrm{X}^{2}=1.7 ; \mathrm{p}=0.43$.

Although J-CTO score was initially proposed to predict the lesion complexity based on wire crossing through the CTO body within $30 \mathrm{~min}$, due to its simplicity it has been widely used in many cath labs to predict the likelihood of procedural success rate.
In the context of a CTO-PCI a meticulous review and evaluation of the case both from an angiographic point of view and in terms of risks and benefits balance is extremely important and is something that should be taken into consideration before the procedure. Although the success rate has dramatically increased during the last decade thanks to the improvement of dedicated materials, evolution of techniques and increase in the level of experience [14] is still below that of the non-occluded coronary arteries. Besides, these types of procedures are time and resource consuming and the incidence of major adverse cardiac events although decreasing over time is still higher compared to non-CTO PCIs [14-16]. Therefore, the availability of scores to predict the likelihood of success or failure in CTO-PCIs and to share this information with the referring physicians and as well as with the patient is something that is highly useful and important. Additionally, the simpler and more practical a score is, the more widely and more easily it can be applied.

Among several scores described so far, the PROGRESS-CTO [4] registry analyzed 781 CTO-PCIs in 7 American centers with significant ex-

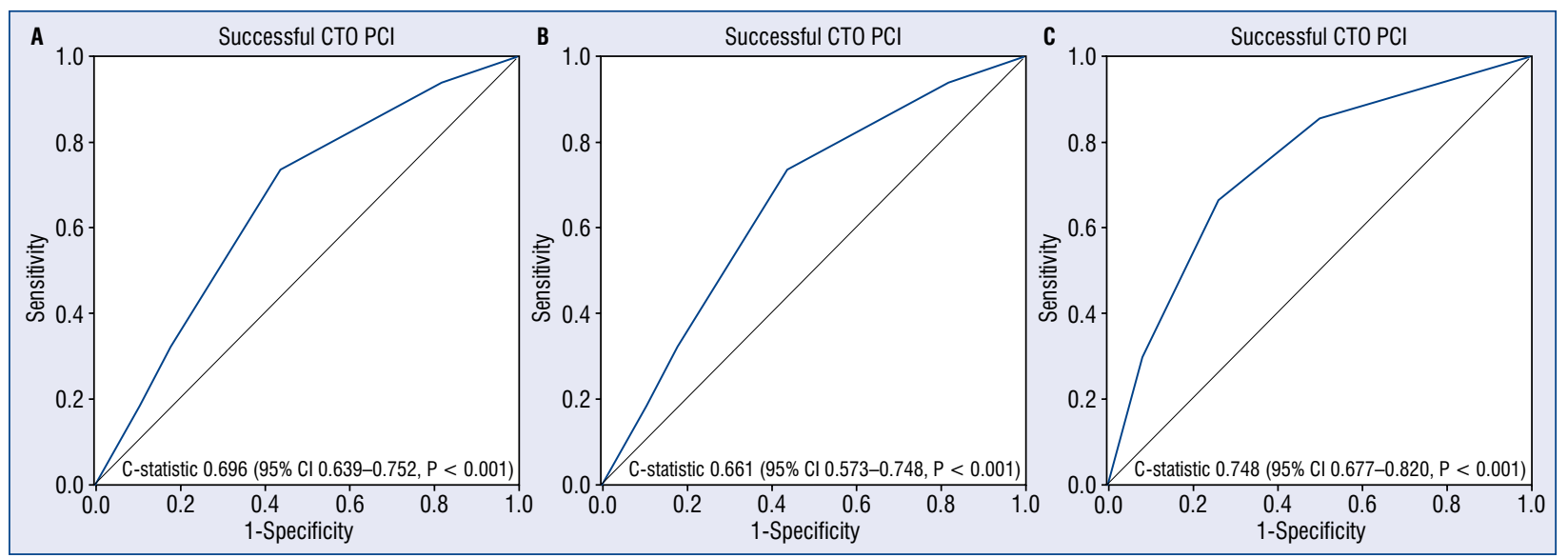

Figure 5. Receiver operator characteristic curve for successful percutaneous coronary intervention (PCI) in the entire cohort (A), in the first block (case 1-200) (B) and in the second block (case 201-526) (C); Cl - confidence interval; CTO - chronic total occlusion. 
pertise in the field of CTO intervention. The score included 4 angiographic characteristics namely no interventional collaterals, proximal cap ambiguity, moderate or severe tortuosity and left circumflex CTO. The model revealed good predictive capacity for procedural success. Furthermore, the score performed similarly to predict technical success as J-CTO score (ROC curve: 0.720 vs. 0.746). It should be mentioned that hybrid algorithm consisting of rapid switch between various CTO crossing strategies [17] has been widely used amongst the operators representing this study and the score included key variables of hybrid strategy such as proximal ambiguity, presence of interventional collaterals and tortuosity, as a surrogate of distal vessel quality. In contrast to PROGRESS CTO score population, our cohort reflects a growing operator experience and low rate of techniques such as antegrade dissection reentry. Moreover, retrograde approach was assimilated to our armamentarium as a higher level of experience was achieved and represented a total rate of $14.4 \%$ in the entire series.

Cl-score [5] combining various angiographic and clinical characteristics namely severe calcified lesion, previous coronary artery bypass grafting, lesion length $\geq 20 \mathrm{~mm}$, previous $\mathrm{MI}$, blunt stump and non-LAD-CTO location found a direct correlation between the probability of procedural failure as the score number increased. However, the ROC curve obtained in this study was 0.68 for $\mathrm{Cl}$-score against 0.60 for J-CTO score.

Karatasakis et al. [18] compared Cl-score, $\mathrm{J}$-CTO, and PROGRESS CTO scores for predicting the success of CTO-PCI and concluded that all scores were inversely associated with technical success with moderate discriminatory capacity (ROC curve 0.691 for $\mathrm{Cl}$ score, 0.682 for J-CTO and 0.647 for PROGRESS CTO score). Another finding of this study was the fact that all three scores tended to perform better in antegrade-only procedures.

CASTLE score derived from 20,000 patients in the EuroCTO Registry [19] with cases submitted by CTO expert operators ( $>50$ cases a year) identified 6 of the following predictors for technical failure: coronary artery bypass grafting history, age ( $\geq 70$ years), stump anatomy (blunt or invisible), tortuosity, occlusion length $\geq 20 \mathrm{~mm}$ and the extent of calcification. Tortuosity in this score was measured in a more complex fashion than how it was assessed in the J-CTO score. The bending was defined as severe when the CTO vessel contained either two or more pre-occlusive bends $>90^{\circ}$ or at least one bend $>120^{\circ}$; and moderate when it contained two bends $>70^{\circ}$ or one bend $>90^{\circ}$. The score showed an area under curve (AUC) for derivation and validation set of 0.66 and 0.68 with slightly higher discriminatory power than J-CTO score which performed an AUC of 0.63 and 0.64 for derivation and validation set respectively. In contrast with the EuroCTO registry, part of the present cohort is represented by less than 50 cases a year and besides, complex techniques such as the retrograde approach were used more frequently in that study than in our cohort (20.2\% vs. $14.4 \%$ ).

On the one hand, some scores incorporate new variables unfounded in the previous scoring systems, which can be explained by the fact that new scores highlight the variety in approaches to CTO-PCI. For instance, ORA score (ostia location, Rentrop grade $<2$, age $\geq 75$ years) described by Galassi et al. [20] reflects the operator experience in hybrid and retrograde approach. On the other hand, the appearance of common variables between different scoring systems is something that contributes to the validation of the previously published scores [21].

Compared to J-CTO registry, the present cohort was more represented by intermediate lesions (35\% vs. $24.9 \%$ ) and less by difficult and especially by very difficult procedures $(21.7 \%$ vs. $28 \%$ and $16.2 \%$ vs. $27.4 \%$ ), respectively. Again, retrograde approach was more frequently attempted in the Japanese series than in the present cohort $(25.7 \%$ vs. $14.4 \%)$.

Recently Karacsonyi et al. [22] compared predictive capacity for CTO technical success of J-CTO, PROGRESS CTO and EUROCTO CASTLE score in 30 high-volume and experienced centers, and concluded that all three scores perform moderately well in predicting technical success with J-CTO score having the best overall performance.

Considering that our series comprises operator skill ranging from a lower to higher level of expertise throughout time that differs in some way to other studies, the applicability of J-CTO score as a practical model was something we considered worthy of scrutiny. Our findings suggest that the score has a moderate performance during the first steps of the learning curve in CTO-PCIs and shows a good predictive power as a higher level of experience was attained.

The availability of a simple and practical score such as J-CTO score can help the operators estimate the level of difficulty of each case in a more objective way, enabling a better case selection and helping to design an adequate strategy before 
tackling the CTO. While seasoned operators can achieve a high success rate performing very complex CTO-PCI [23] less experienced interventionists should ask for counseling or proctoring for more unfavorable cases according to the scoring system. Proctoring in some cases could be highly advisable if the level of complexity surpasses the operator experience. Information about the complexity of cases and success likelihood should be shared in a transparent fashion with referring physicians and with the patient.

\section{Limitations of the study}

This is a single center study with growing experience in the CTO-PCI and with improvement in procedural success over time. The introduction of complex techniques has been gradual and progressive so the external validation of this study is limited to centers with similar operator expertise and comparable strategies. Although a careful review of J-CTO variables was accomplished by two observers with good inter-observer concordance, some bias cannot be ruled out since the ideal scenario would be the assessment of the variables by a core lab uninformed of the results of the procedure.

\section{Conclusions}

The present study reveals that J-CTO score can be applied for the prediction of PCI success rate in a center with growing experience in this field of intervention. Although the predictive power score for the overall cohort was acceptable, the model predicted better as the level of operator experience increased over time.

\section{Conflict of interest: None declared}

\section{References}

1. Yamane M. Current percutaneous recanalization of coronary chronic total occlusion. Revista Española de Cardiología (English Edition). 2012; 65(3): 265-277, doi: 10.1016/j.rec.2011.12.001.

2. Azzalini L, Carlino M, Bellini B, et al. Long-term outcomes of chronic total occlusion recanalization versus percutaneous coronary intervention for complex non-occlusive coronary artery disease. Am J Cardiol. 2020; 125(2): 182-188, doi: 10.1016/j. amjcard.2019.10.034, indexed in Pubmed: 31759516.

3. Werner GS, Martin-Yuste V, Hildick-Smith D, et al. EUROCTO trial investigators. A randomized multicentre trial to compare revascularization with optimal medical therapy for the treatment of chronic total coronary occlusions. Eur Heart J. 2018; 39(26): 2484-2493, doi: 10.1093/eurheartj/ehy220, indexed in Pubmed: 29722796.
4. Christopoulos G, Kandzari DE, Yeh RW, et al. Development and Validation of a Novel Scoring System for Predicting Technical Success of Chronic Total Occlusion Percutaneous Coronary Interventions: The PROGRESS CTO (Prospective Global Registry for the Study of Chronic Total Occlusion Intervention) Score. JACC Cardiovasc Interv. 2016; 9(1): 1-9, doi: 10.1016/j. jcin.2015.09.022, indexed in Pubmed: 26762904.

5. Alessandrino G, Chevalier B, Lefèvre T, et al. A clinical and angiographic scoring system to predict the probability of successful first-attempt percutaneous coronary intervention in patients with total chronic coronary occlusion. JACC Cardiovasc Interv. 2015; 8(12): 1540-1548, doi: 10.1016/j.jcin.2015.07.009, indexed in Pubmed: 26493246.

6. De Jin C, Kim MH, Kim SJ, et al. Predicting successful recanalization in patients with native coronary chronic total occlusion: the Busan CTO Score. Cardiology. 2017; 137(2): 83-91, doi: 10.1159/000455824, indexed in Pubmed: 28171874.

7. Morino $\mathrm{Y}$, Abe M, Morimoto T, et al. Predicting successful guidewire crossing through chronic total occlusion of native coronary lesions within 30 minutes. JACC: Cardiovasc Interv. 2011; 4(2): 213-221, doi: 10.1016/j.jcin.2010.09.024.

8. Nombela-Franco L, Urena M, Jerez-Valero M, et al. Validation of the J-chronic total occlusion score for chronic total occlusion percutaneous coronary intervention in an independent contemporary cohort. Circ Cardiovasc Interv. 2013; 6(6): 635-643, doi: 10.1161/CIRCINTERVENTIONS.113.000447, indexed in Pubmed: 24254710.

9. Young MN, Secemsky EA, Kaltenbach LA, et al. Examining the operator learning curve for percutaneous coronary intervention of chronic total occlusions. Circ Cardiovasc Interv. 2019; 12(8): e007877, doi: 10.1161/CIRCINTERVENTIONS.119.007877, indexed in Pubmed: 31416356.

10. Galassi AR, Werner GS, Boukhris M, et al. Percutaneous recanalisation of chronic total occlusions: 2019 consensus document from the EuroCTO Club. EuroIntervention. 2019; 15(2): 198-208, doi: 10.4244/EIJ-D-18-00826, indexed in Pubmed: 30636678.

11. Mohandes M, Guarinos J, Rodríguez J, et al. Marcadores angiográficos negativos en la intervención coronaria percutánea de las oclusiones totales crónicas. Archivos de Cardiología de México. 2018; 88(2): 93-99, doi: 10.1016/j.acmx.2017.01.008.

12. Morino Y, Kimura T, Hayashi Y, et al. In-Hospital outcomes of contemporary percutaneous coronary intervention in patients with chronic total occlusion. JACC: Cardiovasc Interv. 2010; 3(2): 143-151, doi: 10.1016/j.jcin.2009.10.029.

13. Thygesen K, Alpert J, Jaffe A, et al. Fourth universal definition of myocardial infarction (2018). Eur Heart J. 2018; 40(3): 237-269, doi: 10.1093/eurheartj/ehy462.

14. Sathananthan J, Džavík V. Coronary intervention for chronic total occlusion: current indications and future directions. Coron Artery Dis. 2017; 28(5): 426-436, doi: 10.1097/ MCA.0000000000000490, indexed in Pubmed: 28692460.

15. Hirai K, Sakakura K, Watanabe Y, et al. Determinants of high device cost in current percutaneous coronary interventions. Cardiovasc Revasc Med. 2018; 19(5 Pt B): 607-612, doi: 10.1016/j. carrev.2017.12.007, indexed in Pubmed: 29358042.

16. Brilakis ES, Banerjee S, Karmpaliotis D, et al. Procedural outcomes of chronic total occlusion percutaneous coronary intervention: a report from the NCDR (National Cardiovascular Data Registry). JACC Cardiovasc Interv. 2015; 8(2): 245-253, doi: 10.1016/j.jcin.2014.08.014, indexed in Pubmed: 25700746. 
17. Brilakis ES, Grantham JA, Rinfret S, et al. A percutaneous treatment algorithm for crossing coronary chronic total occlusions. JACC Cardiovasc Interv. 2012; 5(4): 367-379, doi: 10.1016/j. jcin.2012.02.006, indexed in Pubmed: 22516392.

18. Karatasakis A, Danek BA, Karmpaliotis D, et al. Comparison of various scores for predicting success of chronic total occlusion percutaneous coronary intervention. Int J Cardiol. 2016; 224: 50-56, doi: 10.1016/j.ijcard.2016.08.317, indexed in Pubmed: 27611917.

19. Szijgyarto Z, Rampat R, Werner GS, et al. Derivation and Validation of a Chronic Total Coronary Occlusion Intervention Procedural Success Score From the 20,000-Patient EuroCTO Registry: The EuroCTO (CASTLE) Score. JACC Cardiovasc Interv. 2019; 12(4): 335-342, doi: 10.1016/j.jcin.2018.11.020, indexed in Pubmed: 30711551.

20. Galassi AR, Boukhris M, Azzarelli S, et al. Percutaneous coronary revascularization for chronic total occlusions: a novel predictive score of technical failure using advanced technologies.
JACC Cardiovasc Interv. 2016; 9(9): 911-922, doi: 10.1016/j. jcin.2016.01.036, indexed in Pubmed: 27085580.

21. Karatasakis A, Danek BA, Brilakis ES. Scoring systems for chronic total occlusion percutaneous coronary intervention: if you fail to prepare you are preparing to fail. J Thorac Dis. 2016; 8(9): E1096-E1099, doi: 10.21037/jtd.2016.08.20, indexed in Pubmed: 27747074.

22. Karacsonyi J, Stanberry L, Alaswad K, et al. Predicting technical success of chronic total occlusion percutaneous coronary intervention: comparison of 3 scores. Circ Cardiovasc Interv. 2021; 14(1): e009860, doi: 10.1161/CIRCINTERVENTIONS.120.009860, indexed in Pubmed: 33423537.

23. Christopoulos G, Wyman RM, Alaswad K, et al. Clinical utility of the Japan-chronic total occlusion score in coronary chronic total occlusion interventions: results from a multicenter registry. Circ Cardiovasc Interv. 2015; 8(7): e002171, doi: 10.1161/CIRCINTERVENTIONS.114.002171, indexed in Pubmed: 26162857. 